\title{
Aplicação dos sistemas de informação geográfica para definição de estratégias de manejo de bovinos nas pastagens
}

\author{
Adriano Gomes Páscoa ${ }^{1,2}$ e Mateus J.R. Paranhos da Costa ${ }^{1,3}$
}

1 - Grupo ETCO, Grupo de Estudos e Pesquisas em Etologia e Ecologia Animal.

2 - Programa de Pós Graduação em Zootecnia, Doutorado, FCAV/UNESP, Jaboticabal, SP. agpascoa@fcav.unesp.br

3 - Departamento de Zootecnia, FCAV/UNESP, 14880-900, Jaboticabal-SP, Brasil. Pesquisador CNPq. mpcosta@fcav.unesp.br

RESUMO - O estudo de grandes áreas de pastejo, principalmente no que diz respeito ao seu uso pelos animais, é difícil, tanto na forma de amostragem, quanto na maneira de analisar os dados obtidos. Nesse contexto o Sistema de Informação Geográfica (SIG) surge como ferramenta útil, capaz de viabilizar estudos ecológicos e comportamentais associados ao uso do espaço e dos recursos nele alocados. Dentre as ferramentas principais de um SIG com importância para o estudo de ecologia de pastagens, podemos citar a determinação de áreas de uso e trilhas, além das sobreposições, regressões e multi-regressões de mapas (compondo por exemplo: áreas de pastejo e malhadouros, características do solo, concentrações espaciais de nutrientes nas forragens, etc.). Por ser uma ferramenta de modelagem espacial computadorizada nos permite compreender e analisar de maneira mais adequada, os fenômenos de distribuição geográfica muitas vezes imperceptível nas coletas de dados convencionais. A distribuição dos animais em uma área qualquer resulta de ações combinadas de forças internas ao grupo social (por exemplo, hierarquia de dominância, liderança e formação de subgrupos) e externas a ele, como por exemplo, o posicionamento de bebedouros, sombras e cercas, que podem atuar como condicionadores de pastejo. Assim, com o entendimento do complexo arranjo do ambiente de pastagem com o uso do SIG, incluindo os fatores bióticos (tipos de vegetação, por exemplo) e abióticos (relevo), é possível desenvolver estratégias de manejo de forma a modular o comportamento dos animais, reduzindo os problemas que podem causar degradação nos recursos deste ambiente. Porém, a escolha do melhor conjunto de técnicas de análise dentro do SIG e de suas possibilidades de uso ainda está por vir, principalmente no que diz respeito às suas aplicações às condições e necessidades brasileiras.

Palavras-chaves: cerca, cocho, comportamento, distribuição de recursos, sombra, trilhas, uso do espaço

\section{Applying the geografic information systems to define cattle management of pastures}

\begin{abstract}
The study of great grazing areas, mainly so that concerns the use for the animals, it is difficult, so much in the sampling form, as in the way to analyze the obtained data. In that context the Geographical Information System (GIS) it appears as useful tool, capable to make possible ecological and behavior studies associated to the use of the space and of the resources in him allocated. Among the main tools of a GIS with importance for the study of ecology of pastures, we can quote the determination of use areas, trails and overlay, regressions and multiregressions of maps (for instance: grazing areas, soil stains, resting places, space concentrations of nutrients in the forages, etc.). For being a tool of computerized spatial modeling, allows us to understand and to analyze in a more appropriate way, the phenomena of geographical distribution many times imperceptible in the collections of conventional data. The distribution of the animals in any area results from combined actions of internal forces to the social group (for instance, dominantly hierarchy, leadership and subgroups formation) and external to him, as for instance, the water bunker positioning, shadows and fences, that can act as conditioning of grazing. Like this, with the understanding of the compound arrangement of the pasture atmosphere with the use of GIS, including the biotic (vegetation types, for instance) and non- biotic factors(relief), it is possible to develop strategies of form handling to modulate the behavior of the animals, reducing the problems that can cause degradation in the resources of this adapts. However, the choice of the best group of analysis techniques inside of GIS and the possibilities of use it is still for coming, mainly in that concerns their applications to the Brazilian conditions and needs.
\end{abstract}

Key Words: behavior, distributions, feed bunker, fence, resource, share, space use, trails 
Introdução

Um dos caminhos para promover o aumento na produção de carne sem ampliar as áreas de pastagens é a melhoria das mesmas. Isto pode ser alcançado com técnicas mais adequadas de manejo, cuja implementação depende de um melhor entendimento dos mecanismos envolvidos e dos limites que devem ser respeitados para o uso sustentável das pastagens.

Segundo Adler et al. (2001) o estudo de como o pastejo pode alterar a heterogeneidade espacial da vegetação tem importância teórica e prática; se o pastejo altera a estrutura espacial de um ecossistema, ele gera importantes conseqüências nas variadas funções deste mesmo ecossistema. Na prática (e sob uma perspectiva de manejo) um importante tema é a relação entre biodiversidade e heterogeneidade espacial. O pastejo parece influenciar a diversidade de plantas em muitos ecossistemas (Michunas \& Lauenroth, 1993), porém não está claro se mudanças no padrão espacial de pastejo direcionam esse efeito e nem explica porque o pastejo ora aumenta, ora diminui a heterogeneidade (Adler et al., 2001); por exemplo, o comportamento de pastejo dos bovinos não sendo uniforme, causa sub-pastejo em algumas áreas e superpastejo em outras (Goulart, 2006).

Quando esse tipo de informação é mensurado e analisado com a perspectiva de uso de uma estatística não espacial, ela corresponde à variabilidade espacial, mas quando tanto as mensurações quanto as análises levam em conta a espacialização (sendo referenciadas ou georreferenciada) ela corresponde ao padrão espacial (Adler et al., 2001). O autor propôs ainda uma definição operacional para dependência espacial, caracterizando-a como a relação entre valores de uma variável observada em diferentes localizações.

Nesse contexto, surgem os métodos que compõem o Sistema de Informação Geográfica, que pode ser definido como um poderoso conjunto de ferramentas para a coleta, armazenagem, correção, transformação e apresentação de dados espacializados no globo terrestre (georreferenciados) ou em uma área específica, quando somente referenciado (Burrough \& McDonnell, 1998). Este sistema tem sido aplicado na modelagem do comportamento de pastejo em ambientes heterogêneos, emergindo como uma importante

๑) 2007 Sociedade Brasileira de Zootecnia ferramenta no entendimento das relações nos ecossistemas de pastagens (Parson et al., 2001).

Os SIGs consistem em sensoriamento remoto ou local (coleta de dados), análises geostatísticas, interpolação de dados, confecção de mapas e interpretação dos mapas gerados. Segundo Clark (1979) a geo-estatística é a técnica que melhor se enquadra para descrever dados amostrais que são espacialmente correlacionados; ela utiliza o método de análise de semivariograma, o qual define o tipo e a forma de associação espacial (Vieira et al., 1983) e da interpolação pela krigagem - que é um método geoestatístico utilizado para estimar valores em locais não amostrados (Journel \& Huijbregts, 1978). A interpolação é importante quando precisamos relacionar superfícies com resoluções, tamanhos amostrais ou orientação diferentes (Burrough \& McDonnell, 1998). Essa característica é especialmente importante para estudos em que a coleta de dados é difícil de ser espacialmente homogeneizada (principalmente em grandes áreas).

\section{Situação atual da aplicação do SIG em estudos com bovinos}

O comportamento de bovinos em pastejo, relativo às suas preferências por determinadas áreas, pode ser compreendido por algumas escolhas: seleção de forragens em função de suas alturas (Black \& Kenney, 1984; Arnold, 1987; Bazely, 1990), ou das variações nos teores de nutrientes (Bazely, 1990; Langvatn \& Hanley, 1993; Wallisdevries \& Schippers, 1994), ou ainda a seleção de áreas não contaminadas com placas de fezes (Dohi et al., 1991; Hutchings et al., 1998; Pascoa, 2005). Outros autores indicaram que os principais mecanismos de escolha são relacionados com a disponibilidade e distribuição de certos recursos, principalmente a água, mas também os minerais, tanto os encontrados nas plantas quanto em suplementos (Adler et al., 2001; Ganskopp, 2001). Há ainda outros fatores que podem ser apontados como determinantes na definição de sítios e estações de pastejo, dentre eles: relevo (Ganskopp \& Vavra, 1987, Bailey, 2005), fornecimento e alocação de suplementos energéticos e protéicos (Bailey et al., 2001), composição botânica e qualidade da forragem (Bailey et al., 1996), fatores que favorecem a termorregulação 
(como sombra e vento, por exemplo) (Senft et al., 1985; Stuth, 1991) e padrões do comportamento social (Penning et al., 1993; Thouless, 1990; Carvalho et al., 1999). Apesar de tantas variáveis passíveis de influenciar no padrão de pastejo dos bovinos, não existem ainda pesquisas que as interrelacionem.

Possivelmente um pesquisador ficaria louco se tentasse colocar em uma única equação (e aqui não estamos pensando apenas na análise como também na maneira mais adequada de coletar tantos dados em tão pouco espaço de tempo), todas as variáveis capazes de mudar a escolha dos animais em uma área de pastejo. Poucos são os trabalhos nessa área e a maioria deles agrupa as variáveis duas a duas ou aplica apenas as correlações simples para tentar explicar o uso das áreas (Ganskopp et al., 2000, Ganskopp, 2001; Goulart, 2006).

A criação de um passo a passo para a confecção de modelos, onde as variáveis sejam agrupadas por características ou funções desempenhadas no processo de escolha dos animais faz-se, portanto, necessária.

\section{Nossa proposta de trabalho}

Primeiramente temos que entender como os bovinos enxergam a sua casa. Dicas visuais são inicialmente importantes, pois orientam o início do pastejo, diminuem sua latência, aumentam sua duração (Howery et al., 2000). Entre as variáveis que podem ser agrupadas nesse pool (palavra inglesa que tem como um dos significados a combinação de recursos) estariam: a altura e cor da forragem, presença de árvores e de outros grupos animais (geralmente separados por cercas). Outro pool de variáveis poderia compreender aspectos físicos da pastagem, como: tamanho do pasto, relevo, posição de cercas, aguadas e cochos de suplementação, além da disposição de trilhas e malhadouros (lugares onde o gado costuma dormir em lotes ou se reunir para descanso e ruminação) e agregação de fezes. Ainda um terceiro grupo poderia ser formado por características físicoquímicas da forrageira, tais como: concentração de FDN, FDA, lignina e proteína, além da porcentagem de matéria seca e matéria verde e disponibilidade da forrageira. E um quarto e último grupo fatores relacionados ao controle da intensi- dade com que os animais manifestam seus comportamentos, como chuvas, temperatura, umidade, radiação, etc.

Para exemplificar a utilização do conjunto de métodos com a aplicação do SIG foi realizada uma re-análise de nossos dados (Pascoa, 2005), em estudo conduzido em um piquete de Tifton 85 (Cynodon dactilon, L. Pers) com aproximadamente $3.000 \mathrm{~m}^{2}$. O piquete dispunha de bebedouro e cocho para suplementação mineral e também havia uma área com alta concentração de material orgânico, produto do acúmulo de fezes depositadas ao longo dos anos no perímetro de um cocho de suplementação, que foi retirado da área 12 meses antes do início de nosso estudo. Essa área, com formato ovalado, tinha aproxi-madamente $90 \mathrm{~m}^{2}$ e se localizava em um dos cantos do piquete. Para a coleta de dados foram definidos quadrantes no piquete, sendo 24 quadrantes para a amostragem de forragem (com 12x12 m) e 384 quadrantes para a contagem de fezes (com $3 \times 3 \mathrm{~m}$ cada quadrante). O período de estudo foi de um ano e meio, com início em janeiro de 2003 e término em setembro de 2004.

Foram utilizadas 10 novilhas da raça Nelore do plantel da Estação Experimental de Zootecnia de Sertãozinho, com idade média de 30 meses, com peso vivo médio de $416 \mathrm{~kg}$ e um animal que variava conforme a época do ano entre um touro, utilizado no período de monta da fazenda, e uma vaca ambos com peso médio de $598 \mathrm{~kg}$ para que fosse mantida a mesma lotação ao longo do estudo. Ao início de cada ciclo de ocupação, os animais eram identificados com números pintados nos flancos e garupas. Essa marcação foi necessária para avaliar o comportamento individual de pastejo de cada animal.

Para o propósito deste artigo foi feita uma análise de correlação multi-variada, no programa de SIG, IDRISI v.15.00 (Clark, 2006). Essa análise levou em conta a importância das variáveis independentes na composição da dependente, mas baseada na distribuição espacial das mesmas (quanto maior a distância menor o peso relativo da variável). Os tempos de pastejo em cada um dos quadrantes do piquete foram avaliados com base nas características físico-químicas da forragem em cada quadrante, sendo encontrada a seguinte equação (equação 1):

๑ 2007 Sociedade Brasileira de Zootecnia 


\section{PASTEJO = 22,14 - 0,16DCOS - 0,30MS + 1,29PB + 0,001DISP + 0,12FDA - 0,70FDN + 0,10LIG - 0,0003PV;}

onde:

DCOS = distância de uma dada área em relação a área onde havia um antigo cocho de suplementação;

MS $=\quad \%$ de matéria seca do capim no ciclo 1 e na entrada dos animais no piquete;

$\mathrm{PB}=$ concentração de proteína bruta na forragem no ciclo 1 e na entrada dos animais no piquete;

DISP $=$ disponibilidade de forragem em gramas de matéria seca por $\mathrm{m}^{2}$ (ciclo 1, entrada);

$\mathrm{FDA}=\%$ de fibra em detergente ácido da forragem no ciclo 1 e na entrada dos animais no piquete;

$\mathrm{FDN}=\%$ de fibra em detergente neutro da forragem no ciclo 1 e na entrada dos animais no piquete;

$\mathrm{LIG}=\quad \%$ de lignina na forragem no ciclo 1 e na entrada dos animais no piquete; $\mathrm{PV}=\quad$ disponibilidade de forrageira em gramas de peso verde por $\mathrm{m}^{2}$ (ciclo 1, entrada). Nessa análise, foi encontrado um coeficiente de determinação $\left(r^{2}\right)$ igual a 0,633 .

Quando foram agrupadas as variáveis das características estruturais do piquete foi obtida a seguinte equação 2 :

\section{PASTEJO = 6,45 +0,08DBEB -0,04DCER - 0,25DCOS -0,06DPORT +0,02DSAL $+1,26 \mathrm{FEZD1}$;}

onde:

$\mathrm{DBEB}=$ distância de um ponto no piquete até o bebedouro (em metros);

DCER = distância de um ponto no piquete até a cerca elétrica (m);

DCOS = é a distância de uma área onde se localizava um antigo cocho;

DPORT = distância de um ponto no piquete até uma das porteiras $(\mathrm{m})$;

DSAL = distância de um ponto no piquete até o cocho com suplemento mineral (m) e
FEZD1 = concentração de placas de fezes no ciclo 1 e no primeiro dia de pastejo.

Nessa análise encontramos um coeficiente de determinação menor que na anterior $\left(r^{2}=0,601\right)$, o que nos levou a reformular os fatores que compõem as equações para predição do uso da pastagem pelos animais. Na primeira equação, peso verde e disponibilidade de forragem não mostraram ter grande influência no processo de decisão dos animais na escolha do(s) local(is) de pastejo. Esse pode ser um importante critério na escolha das variáveis que irão compor a equação final. Na segunda, a decisão seguiu outro critério: o do bom-senso: a distância da porteira seria um ponto importante para a decisão de um animal ao pastejo? Caso não haja nenhum recurso fora do piquete, não! A concentração de fezes foi também retirada da equação, baseado na expectativa de que no primeiro dia de pastejo não existiam placas de fezes no piquete (visto que houve um período de descanso antes do início do estudo de um ano).

Com estas exclusões e combinando as duas equações obtivemos a equação 3 , como segue:

\section{PASTEJO = 35,90 -0,08DB -0,12DCE - 0,24DCO -0,13DSAL -0,003FDA -0,57FDN $+0,11$ LIG -0,38MS +1,42PB;}

onde:

$\mathrm{DB}=\quad$ distância de um ponto no piquete até o bebedouro (em metros);

$\mathrm{DCE}=$ distância de um ponto no piquete até a cerca elétrica $(\mathrm{m})$;

$\mathrm{DCO}=$ é a distância de uma área onde se localizava um antigo cocho;

$\mathrm{DSAL}=$ distância de um ponto no piquete até o cocho de mineralização $(\mathrm{m})$;

$\mathrm{FDA}=\%$ de fibra em detergente ácido da forragem (ciclo 1, entrada);

$\mathrm{FDN}=\%$ de fibra em detergente neutro da forragem (ciclo 1, entrada);

$\mathrm{LIG}=\quad \%$ de lignina na forragem (ciclo 1 , entrada);

MS $=\quad \%$ de matéria seca do capim (ciclo 1, entrada) e

$\mathrm{PB}=\quad$ concentração de proteína bruta na forragem (ciclo 1, entrada). Neste caso o coeficiente de determinação 
foi maior $\left(r^{2}=0,658\right)$. Nesse estudo não foram levados em consideração indicadores visuais, pois não havia árvores no piquete e não foram avaliadas a altura nem a cor da forragem.

Uma estratégia interessante para o melhor entendimento do papel dessas variáveis na definição do pastejo é proceder à análise de resíduos da regressão pelo SIG (Eastman, 1997); com ela é possível gerar mapas de resíduos, que caracterizam, dentro do piquete, as áreas onde a equação de regressão gera valores superestimados (valores negativos), subestimados (valores positivos) ou valores reais de pastejo, como apresentado na Figura 1, sendo que as áreas em vermelho caracterizam que o pastejo foi subestimado e as áreas em azul superestimado, as áreas em preto representam aquelas em que o pastejo foi bem representado pelo modelo de regressão apresentado na equação 3 .

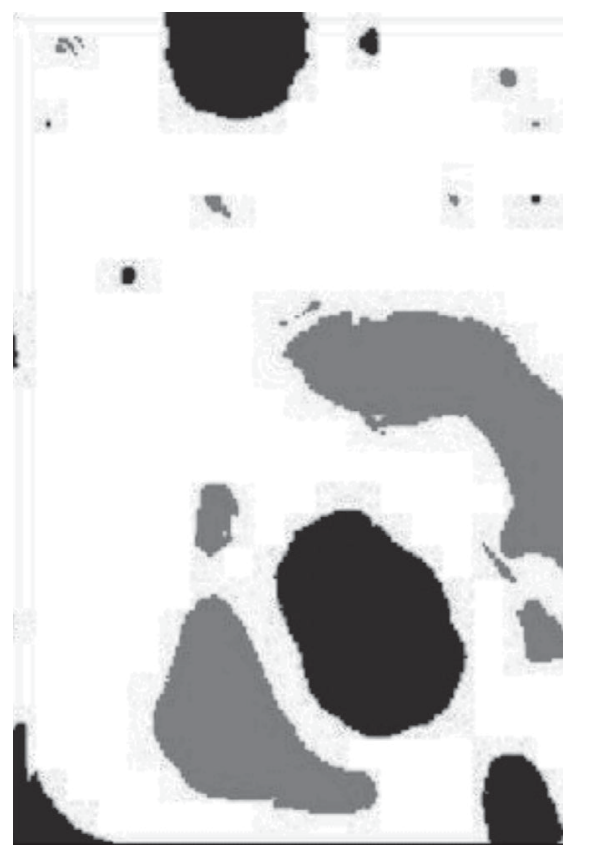

Figura 1 - Mapa dos resíduos de regressão do pastejo dado pela equação 3: as áreas em preto caracterizam áreas em que o pastejo pode ter sido subestimado, as áreas em cinza aquelas em que pode ter sido superestimado e em branco são as áreas em que o pastejo foi bem representado pelo modelo de regressão.

Com este tipo de análise fica mais fácil identificar quão eficiente é um determinado modelo para explicar distribuições espaciais (de qualquer coisa ou atividade) e como o nível de eficiência das estimativas geradas por este modelo varia dentro da própria área de estudo (Eastman, 1997). Por exemplo, na Figura 1 foram identificadas que as áreas próximas do cocho de sal (área em vermelho na parte de cima da Figura 1), ao bebedouro (área em vermelho na parte de baixo e à esquerda da Figura 1) e ao local onde havia um cocho de suplementação (área em vermelho na parte de baixo e à direita, caracterizando um formato de elipse, na Figura 1) foram áreas com maior pastejo, sendo que os valores subestimados pela equação 3. Já as áreas caracterizadas pela cor azul (localizadas no entorno da área do antigo cocho) tiveram seu uso para o pastejo superestimado, o que era esperado, dado a preferência de pastejo em área vizinha. Convém lembrar que neste exemplo não foram consideradas as características de cor e de altura do capim, é provável que se estas variáveis fossem incluídas na equação poderíamos obter resultados distintos, pois era evidente (mesmo sem ter tomado medidas objetivas) que a forragem presente na área onde havia o cocho de suplementação era mais verde e mais alta do que o restante da forragem em outras áreas do piquete.

As possibilidades são enormes, talvez esta seja a razão de tão poucos trabalhos que contemplem a interação entre um número de variáveis quase que infinitas (podemos passar dias apenas criando escores e formas diferentes de medir uma mesma variável). Todavia, simplificar não é a solução. Quando minimizamos o número de variáveis em cada análise corremos o risco de não encontrarmos respostas às nossas questões ou, quando estas são encontradas podem ser insatisfatórias.

Tomemos novamente como exemplo o estudo acima descrito. Assumindo que levaríamos em conta apenas uma variável para explicar a escolha pelos animais de certos locais para pastejo, nesses casos em geral encontramos coeficientes de determinação menores (Tabela 1) do que aqueles obtidos em análises de regressão múltipla, que foi de $88,09 \%$ quando considerado o conjunto destas variáveis em uma análise de regressão múltipla.

Tudo isto parece muito óbvio, mas geralmente não levado em conta em muitas das pesquisas que tratam deste tema. Além disso, é fácil perceber que o valor do coeficiente de determinação obtido com

๑ 2007 Sociedade Brasileira de Zootecnia 
a regressão múltipla não é uma somatória dos valores obtidos com as regressões simples. Isto porque há uma série de interações entre as diversas variáveis, que acabam se sobrepondo de modo que os pesos de cada uma delas individualmente são diferentes de quando são analisadas em conjunto. Essa análise espacial só é possível graças a geostatística e ao Sistema de Informação Geográfica.

Tabela 1 - Coeficientes de determinação em análises de regressão simples onde a variável dependente é a porcentagem de tempo de pastejo em determinado quadrante e as variáveis independentes são as distâncias do bebedouro, cercas, área de um antigo cocho e sal, concentrações de FDA, FDN, Lignina e PB, além da porcentagem de matéria seca, consideradas sempre uma-a-uma.

\begin{tabular}{lc}
\hline Variável & $\begin{array}{c}\text { Coeficiente de determi } \\
\text { nação encontrado }\end{array}$ \\
\hline Distância do bebedouro & $1,35 \%$ \\
Distância das cercas & $3,19 \%$ \\
Distância do antigo cocho & $54,44 \%$ \\
Distância do sal & $30,62 \%$ \\
FDA & $21,22 \%$ \\
FDN & $64,51 \%$ \\
Lignina & $19,69 \%$ \\
Matéria Seca & $51,43 \%$ \\
Proteína Bruta & $63,19 \%$ \\
\hline
\end{tabular}

Estudos longitudinais também podem fazer uso de SIG, por exemplo, na Figura 2 fica bem caracterizado que a variação na importância da distância do bebedouro em relação ao local escolhido para pastejo é extremamente dependente da época em que o pastejo ocorre, sendo claro que quanto menor a precipitação maior se torna à importância da distância do bebedouro para a escolha do local de pastejo.

Há outros tipos de análises passíveis de serem realizadas com SIG, por exemplo, o método pode ser aplicado na caracterização de padrão de uso do espaço, como exemplificado na Figura 3, que mostra a dispersão de placas de fezes em uma área de pastagem; esta figura foi gerada por um dos programas de geostatística (GS+), que permite gerar informações para espaços não amostrados, usando a técnica de krigagem para interpolação (já descrita neste artigo).

Com o SIG podemos estimar a área total ocupada por cada uma das classes de concentração de placas de fezes, gerando números (como os apresentados na Tabela 2), que facilitam a análise do mapa de dispersão acima. Aliando estas informações ao do posicionamento de estruturas na pastagem (porteiras, bebedouros, cochos, sombras e etc.) é possível definir estratégias mais adequadas para o manejo, de forma que a aplicação do SIG passa a ter um papel importante na tomada de decisões, como já caracterizado por Burrough \& McDonnel (1998).

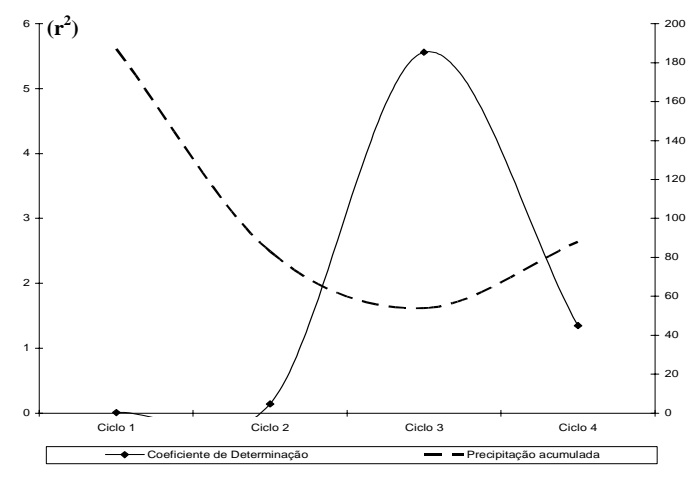

Figura 2 - Coeficientes de determinação para distância do bebedouro em relação ao local de pastejo e valores de precipitação acumuladas nos quatro ciclos de ocupação.

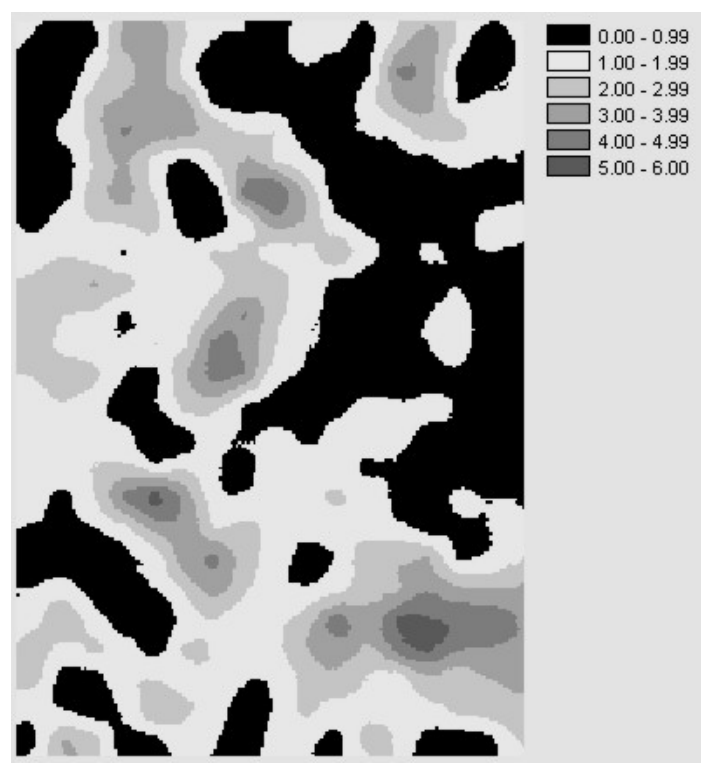

Figura 3 - Mapa de dispersão de placas de fezes em uma área de pastagem, onde os tons mais escuros representam as áreas de maior concentração de placas de fezes e vice-e-versa (número de placas $/ \mathrm{m}^{2}$ ).

Resumindo, modificações do padrão de pastejo dos animais, induzidas por qualquer característica com dependência de distribuição 
Tabela 2 - Área $\left(\mathrm{em} \mathrm{m}^{2}\right)$ e porcentagem da área ocupada por classes de concentração de placas de fezes.

\begin{tabular}{ccc}
\hline $\begin{array}{c}\text { Classe de concentração } \\
\text { de placas de fezes }\end{array}$ & $\begin{array}{c}\text { Área }\left(\mathbf{e m ~}^{2}\right) \text { ocupada por } \\
\text { cada classe }\end{array}$ & $\begin{array}{c}\text { Porcentagem } \\
\text { da área }\end{array}$ \\
\hline $1(0-0,99$ placas $)$ & 356.70 & $11,95 \%$ \\
$2(1-1,99$ placas $)$ & 1204.89 & $41,65 \%$ \\
$3(2-2,99$ placas $)$ & 826.40 & $28,25 \%$ \\
$4(3-3,99$ placas $)$ & 340.10 & $11,40 \%$ \\
$5(4-4,99$ placas $)$ & 160.54 & $5,35 \%$ \\
$6(5-5,99$ placas $)$ & 36.28 & $1,20 \%$ \\
\hline
\end{tabular}

espacial, podem interferir no desempenho dos animais (ganho de peso, produção de leite, fertilidade, etc.) e na otimização do uso da pastagem. Desta forma a aplicação dos métodos SIG vem facilitar o trabalho de pesquisadores, produtores e técnicos, proporcionando uma melhor compreensão das relações entre os diversos fatores envolvidos e gerando informações mais confiáveis para as decisões de manejo.

\section{Literatura citada}

ADLER, P.B.; RAFF, D.A.; LAUENROTH, W.K. The effect of grazing on the spatial heterogeneity of vegetation. Oecologia, v.128, p.465-479, 2001.

ARNOLD, G.E. Influence of the biomass, botanical composition and sward height of annual pastures on foraging behaviour of sheep. Journal Applied Ecology, v.24, p.759-772, 1987.

BAILEY, D.W. Identification and creation of optimum habitat conditions for livestock. Rangeland Ecology and Management, v.58, p.109-118, 2005.

BAILEY, D.M.; KRESS, D.D.; ANDERSON, D.C. et al. Relationship between terrain use and performance of beef cows grazing foothill rangeland. Journal of Animal Science, Champaign, v.79, p.1883-1891, 2001.

BAILEY, D.W.; GROSS, J.E.; LACA, E.A. et al. Mechanisms that result in large herbivore grazing distribution patterns. Journal of Range Management, v.49, p.386-400, 1996.

BAZELY, D.R. Rules and cues used by sheep foraging in monocultures. In: HUGHES, R.N. (ed.), Behavioural Mechanisms of Food Selection. Springer, London, p.343-367, 1990.

BLACK, J.L., KENNEY, P.A. Factors affecting diet selection by sheep. II. Height and density of Pasture. Australian Journal Agricultural Research, v.35, p.565-578, 1984.

BURROUGH, P.A.; MCDONNELL, R.A. Principles of Geographical Information Systems, Oxford University Press, Nova York, 333p., 1998.

CARVALHO, P.C.F.; PRACHE, S.; DAMASCENO, J.C. O processo de pastejo: desafios da procura e apreensão da forragem pelo herbívoro. In: Reunião Anual da Sociedade Brasileira de Zootecnia, 36, Porto Alegre, 1999. Anais... Porto Alegre: SBZ, p.253-268, 1999.

CLARK Labs, IDRISI Andes, v.15.00, Clark University, Worcester, 2006

CLARK, I. Pratical geostatistics. London: Applied Science Plubishers, 1979.

DOHI, H.; YAMADA, A.; ENTSU, S. Cattle feeding deterrents emitted from cattle faeces. Journal Chemical Ecology, v.17, p.1197-1203, 1991.

EASTMAN, J.R. IDRISI for windows user's guide version 2.0: Tutorial exercises. Worcester, MA, Clark University, 192p., 1997.
GANSKOPP, D. Manipulating cattle distribution with salt and water in large arid-land pastures: a GPS/GIS assessment. Applied Animal Behavior Science, v.73, p.251-262, 2001.

GANSKOPP, D.; CRUZ, R.; JOHNSON, D.E. Least-effort pathways?: a GIS analysis of livestock trails in rugged terrain, Applied Animal Behaviour Science, v.68, p.179-190, 2000.

GANSKOPP, D.; VAVRA, M. Slope use by cattle, feral horses, deer, and bighorn sheep, Northwest Science, Port Angeles, v.61, n.2, p.74-81, 1987.

GOULART, R.C.D. Mecanismos envolvidos na escolha de locais de pastejo por bovines de corte. Dissertação (Mestrado em Agronomia) - Escola Superior de Agricultura Luiz de Queiroz, Piracicaba. 74p., 2006.

HOWERY, L.D.; BAILEY, D.W.; RUYLE, G.B. et al. Cattle use visual cues to track food locations. Applied Animal Behaviour Science, v.67, p.1-14, 2000.

HUTCHINGS, M.R.; KYRIAZAKIS, I.; ANDERSON, D.H. et al. Behavioural strategies used by parasitised and nonparasitised sheep to avoid ingestion of gastrointestinal nematodes. Animal Science, v.67, p.97-106, 1998.

JOURNEL, A.G. \& HUIJBREGTS, C.J. Mining geostatistics. Academis Press, London, 1978.

LANGVATN, R. \& HANLEY, T.A. Feeding patch choice by red deer in relation to forging efficiency. Oecologia, v.95, p.164-170, 1993.

MILCHUNAS, D.G. \& LAUENROTH, W.K. Quantitative effects of grazing on vegetation and soils over a global range of environments. Ecological Monography, v.63, p.327-366, 1993

PARSONS, A.J.; SCHWINNING, S.; CARRERE, P. Plant growth functions and possible spatial and temporal scaling errors in models of herbivory. Grass Forage Science, v.56, p.21-34, 2001.

PÁSCOA, A.G. Comportamento de bovinos da raça Nelore mantidos em pastagem de Cynodon spp cv Tifton 85: defecação e rejeição da forragem contaminada por fezes. Dissertação (Mestrado em Zootecnia) - Faculdade de Ciências Agrárias e Veterinárias, Universidade Estadual Paulista, Jaboticabal. 50p. 2005.

PENNING, P.D.; PARSONS, A.J.; NEWMAN, J.A. et al. The effects of group size on grazing time in sheep. Applied Animal Behaviour Science, v.37, p.101-109, 1993.

SENFT, R.L.; RITTENHOUSE, L.R.; WOODMANSEE, R.G. Factors influencing patterns of cattle grazing behavior on shortgrass steppe. Journal of Range Management, v.38, p.82-87, 1985

STUTH, J.W. Foraging behaviour. In: HEITSCHMIDT, R.K.; STUTH, J.W.(eds). Grazing management: an ecological perspective. Oregon: Timber Press, p.85-108, 1991.

THOULESS, C.R. Feeding competition between grazing red deer hinds. Animal Behavior, 40, p.105-111, 1990.

VIEIRA, S.R.; HATFIELD, J.L.; NIELSEN, D.R. et al. Geostatistical theory and application to variability of some agronomical properties. Hilgardia, v.51, n.3, p.1-75, 1983.

WALLISDEVRIES, M.F. \& SCHIPPERS, P. Foraging in a landscape mosaic: selection for energy and minerals in freeranging cattle. Oecologia, v.100, p.107-117, 1994.

๑ 2007 Sociedade Brasileira de Zootecnia 